\title{
Oncologic outcomes of segmentectomy compared with lobectomy for clinical stage IA lung adenocarcinoma: Propensity score-matched analysis in a multicenter study
}

\author{
Yasuhiro Tsutani, MD, PhD, ${ }^{a}$ Yoshihiro Miyata, $\mathrm{MD}, \mathrm{PhD},{ }^{\mathrm{a}}$ Haruhiko Nakayama, $\mathrm{MD}, \mathrm{PhD},{ }^{\mathrm{b}}$ \\ Sakae Okumura, MD, PhD, ${ }^{\mathrm{c}}$ Shuji Adachi, $\mathrm{MD}, \mathrm{PhD},{ }^{\mathrm{d}}$ Masahiro Yoshimura, $\mathrm{MD}, \mathrm{PhD},{ }^{\mathrm{e}}$ and \\ Morihito Okada, MD, $\mathrm{PhD}^{\mathrm{a}}$
}

\begin{abstract}
Objective: Our objective was to compare the oncologic outcomes of lobectomy and segmentectomy for clinical stage IA lung adenocarcinoma.

Methods: We examined 481 of 618 consecutive patients with clinical stage IA lung adenocarcinoma who underwent lobectomy or segmentectomy after preoperative high-resolution computed tomography and F-18-fluorodeoxyglucose positron emission tomography/computed tomography. Patients $(n=137)$ who underwent wedge resection were excluded. Lobectomy $(n=383)$ and segmentectomy $(n=98)$ as well as surgical results were analyzed for all patients and their propensity score-matched pairs.
\end{abstract}

\begin{abstract}
Results: Recurrence-free survival (RFS) and overall survival (OS) were not significantly different between patients undergoing lobectomy (3-year RFS, 87.3\% ; 3-year OS, 94.1\%) and segmentectomy (3-year RFS, 91.4\%; hazard ratio [HR], 0.57; 95\% confidence interval [CI], 0.27-1.20; $P=.14 ; 3$-year OS, 96.9\%; HR, 0.49; 95\% CI, $0.17-1.38 ; P=.18)$. Significant differences in clinical factors such as solid tumor size $(P<.001)$, maximum standardized uptake value (SUVmax) $(P<.001)$, and tumor location (side, $P=.005$; lobe, $P=.001$ ) were observed between both treatment groups. In 81 propensity score-matched pairs including variables such as age, gender, solid tumor size, SUVmax, side, and lobe, RFS and OS were similar between patients undergoing lobectomy (3-year RFS, 92.9\%, 3-year OS, 93.2\%) and segmentectomy (3-year RFS, 90.9\%; 3-year OS, $95.7 \%)$.
\end{abstract}

Conclusions: Segmentectomy is suitable for clinical stage IA lung adenocarcinoma, with survivals equivalent to those of standard lobectomy. (J Thorac Cardiovasc Surg 2013;146:358-64)

Segmentectomy for treating small lung cancer has been a topic of debate for a long time. In 1995, the Lung Cancer Study Group conducted a prospective randomized controlled trial comparing limited resection (including segmentectomy and wedge resection) with lobectomy for clinical T1 N0 M0 non-small cell lung cancer (NSCLC). The study concluded that limited resection resulted in higher local recurrence and lower survival. ${ }^{1}$ A recent study from the Surveillance Epidemiology and End Results database showed that lobectomy conferred a significant advantage compared with segmentectomy in stage I NSCLC. ${ }^{2}$ In contrast, several studies reported that the survivals after segmentectomy and

\footnotetext{
From the Department of Surgical Oncology, ${ }^{\mathrm{a}}$ Hiroshima University, Hiroshima; the Department of Thoracic Surgery, ${ }^{b}$ Kanagawa Cancer Center, Yokohama; the Department of Thoracic Surgery, ${ }^{\mathrm{c}}$ Cancer Institute Hospital, Tokyo; and the Departments of Radiology ${ }^{\mathrm{d}}$ and Thoracic Surgery, ${ }^{\mathrm{e}}$ Hyogo Cancer Center, Akashi, Japan.

Disclosures: Authors have nothing to disclose with regard to commercial support.

Received for publication Oct 4, 2012; revisions received Jan 10, 2013; accepted for publication Feb 11, 2013; available ahead of print March 13, 2013.

Address for reprints: Morihito Okada, MD, PhD, Department of Surgical Oncology, Research Institute for Radiation Biology and Medicine, Hiroshima University, 1-2-3-Kasumi, Minami-ku, Hiroshima City, Hiroshima 734-0037, Japan (E-mail: morihito@hiroshima-u.ac.jp).

0022-5223/\$36.00

Copyright (c) 2013 by The American Association for Thoracic Surgery

http://dx.doi.org/10.1016/j.jtcvs.2013.02.008
}

those after lobectomy were similar. ${ }^{3-7}$ However, few reports compare between segmentectomy and lobectomy with matched patient variables affecting survival.

Recently, we ${ }^{8,9}$ reported that solid tumor size, defined as the maximum dimension of the solid component excluding the ground-glass opacity (GGO) component on highresolution computed tomography (HRCT), and maximum standardized uptake value (SUVmax) on [18F]-fluoro-2deoxy-D-glucose positron emission tomography/computed tomography (FDG-PET/CT), are useful for predicting the pathologic invasiveness or prognosis in clinical stage IA lung adenocarcinoma. These preoperative radiologic findings are important when choosing treatment strategies for NSCLC, particularly for lung adenocarcinoma. ${ }^{8,9}$

The purpose of this retrospective study was to compare the oncologic outcomes between lobectomy and segmentectomy in patients with clinical stage IA lung adenocarcinoma, adjusted for preoperative factors including HRCT and FDG-PET/CT findings, to minimize the effect of patient selection bias. Segmentectomy and wedge resection are considerably different procedures for lung cancer; the former can be used to approach hilar lymph nodes and to get sufficient margin, whereas the latter cannot. Therefore, we excluded wedge resection from this study. 


\section{Abbreviations and Acronyms \\ CI $=$ confidence interval \\ FDG-PET/CT $=[18 \mathrm{~F}]$-fluoro-2-deoxy-D-glucose positron emission tomography/ computed tomography \\ GGO = ground-glass opacity \\ HRCT = high-resolution computed tomography \\ NSCLC = non-small cell lung cancer \\ OS $\quad=$ overall survival \\ RFS $\quad=$ recurrence-free survival \\ SUVmax $\quad=$ maximum standardized uptake value}

\section{PATIENTS AND METHODS \\ Patients}

We enrolled 618 patients with clinical T1 N0 M0 stage IA lung adenocarcinoma from 4 institutions (Hiroshima University, Kanagawa Cancer Center, Cancer Institute Hospital, and Hyogo Cancer Center, Japan) between August 1, 2005 and June 30, 2010, to evaluate the significance of FDG-PET/CT. Patients with incompletely resected tumors (R1 or R2) and those with multiple tumors or previous lung operations were not included in the database. The database has been maintained prospectively. The patient data obtained from this multicenter database were retrospectively analyzed in the present study. HRCT and FDG-PET/CT followed by curative $\mathrm{R} 0$ resection were performed for all patients staged according to the TNM Classification of Malignant Tumors, seventh edition. ${ }^{10}$ Mediastinoscopy or endobronchial ultrasonography was not routinely performed because all patients received preoperative HRCT and FDG-PET/CT; HRCT revealed no swelling of mediastinal or hilar lymph nodes and FDG-PET showed no accumulation in these lymph nodes. Sublobar resection was allowed in cases of complete removal of the disease, using the optional procedure instead of lobectomy for a peripheral T1 N0 M0 tumor. The other patients underwent standard lobectomy. All patients who underwent segmentectomy were suitable for lobectomy and all patients who underwent lobectomy were technically suitable for segmentectomy. Patients who had lymph node metastasis pathologically received platinum-based chemotherapy after operation.

The inclusion criteria were preoperative staging determined by HRCT and FDG-PET/CT, curative surgery without neoadjuvant chemotherapy or radiotherapy, and a definitive histopathologic diagnosis of lung adenocarcinoma. The study was approved by the institutional review boards of the participating institutions; the requirement for informed consent from individual patients was waived because the study was a retrospective review of the patient database. Of the 618 patients, 137 who underwent wedge resection were excluded; the remaining 481 were included in this analysis.

\section{HRCT}

Sixteen-row multidetector CT was used to obtain chest images independent of subsequent FDG-PET/CT examinations. For high-resolution images of the tumors, the following parameters were used: $120 \mathrm{kVp}, 200$ $\mathrm{mA}, 1$ - to 2 -mm section thickness, $512 \times 512$-pixel resolution, 0.5 - to 1.0 -second scanning time, a high-spatial reconstruction algorithm with a 20-cm field of view, and mediastinal (level, $40 \mathrm{HU}$; width, $400 \mathrm{HU}$ ) and lung (level, $-600 \mathrm{HU}$; width, $1600 \mathrm{HU}$ ) window settings. GGO was defined as a misty increase in lung attenuation without obscuring the underlying vascular markings. We defined solid tumor size as the maximum dimension of the solid component measured on lung window settings, excluding GGO. ${ }^{8} \mathrm{CT}$ scans were reviewed and tumor sizes were determined by radiologists from each institution.

\section{FDG-PET/CT}

Patients were instructed to fast for at least 4 hours before intravenous injection of 74 to $370 \mathrm{MBq}$ FDG and were then advised to rest for at least 1 hour before FDG-PET/CT scanning. Blood glucose levels were calculated before the tracer injection to confirm a level of more than 150 $\mathrm{mg} / \mathrm{dL} .{ }^{11}$ Patients with blood glucose levels of $150 \mathrm{mg} / \mathrm{dL}$ or more were excluded from the PET/CT imaging. For imaging, Discovery ST (GE Healthcare, Little Chalfont, United Kingdom), Aquiduo (Toshiba Medical Systems Corporation, Tochigi, Japan), or Biograph Sensation 16 (Siemens Healthcare, Erlangen, Germany) integrated 3-dimensional PET/CT scanner was used. Low-dose nonenhanced CT images of 2- to 4-mm section thickness for attenuation correction and localization of lesions identified by PET were obtained from the head to the pelvic floor of each patient according to a standard protocol.

Immediately after CT, PET was performed with the identical axial field of view for 2- to 4-min/table position, depending on the condition of the patient and the scanner performance. An iterative algorithm with CT-derived attenuation correction was used to reconstruct all PET images with a 50-cm field of view. An anthropomorphic body phantom (NEMA NU2-2001, Data Spectrum Corp, Hillsborough, NC) was used to minimize the variations in SUVs among the institutions. ${ }^{12}$ A calibration factor was analyzed by dividing the actual SUV by the gauged mean SUV in the phantom background to decrease interinstitutional SUV inconsistencies; the final SUV used in this study is referred to as the revised SUVmax. ${ }^{13,14}$ When the SUVmax ratio was expressed as the SUVmax of each institute relative to the SUVmax of the control institute, the adjustment of interinstitutional variations in SUV narrowed the range from 0.89-1.24 to $0.97-1.18$. The original SUVmax values were determined by radiologists from each institution.

\section{Follow-up Evaluation}

All patients who underwent lung resection were followed up from the day of surgery. Postoperative follow-up procedures, including a physical examination and chest radiograph every 3 months and chest and abdominal CT examinations every 6 months, were performed for the first 2 years. Subsequently, a physical examination and chest radiograph were performed every 6 months, and a chest CT examination was performed every year.

\section{Statistical Analysis}

Data are presented as numbers (percent) or the median unless otherwise stated. The $\chi^{2}$ test for categorical variables was used to compare frequencies, and Fisher's exact test was applied to small samples in all cohorts. McNemar tests were used to analyze the propensity-matched pair patients. Both $t$ tests and Mann-Whitney $U$ tests were used to compare continuous variables in all cohorts. Wilcoxon tests were used to analyze propensitymatched pair patients. Recurrence-free survival (RFS) was defined as the time from the day of surgery until the first event (relapse or death from any cause) or last follow-up. Overall survival (OS) was defined as the time from the day of surgery until death from any cause or the last follow-up. The Kaplan-Meier method was used to analyze the duration of RFS and OS; the Cox proportional hazard model was used to assess differences in RFS and OS. We applied propensity score matching to balance the assignment of the included patients and to correct for the operative procedure (lobectomy or segmentectomy), which confounded survival calculations. The variables were age, gender, solid tumor size, SUVmax, side, and lobe. Because no segmentectomy was performed for a tumor located at a middle lobe, we excluded patients who underwent middle lobectomy from the scoring for a fair comparison. Each variable was multiplied by 
TABLE 1. Patient characteristics

\begin{tabular}{lccc}
\hline & $\begin{array}{c}\text { Lobectomy } \\
(\mathbf{n}=\mathbf{3 8 3})\end{array}$ & $\begin{array}{c}\text { Segmentectomy } \\
(\mathbf{n = 9 8})\end{array}$ & $\boldsymbol{P}$ value \\
\hline Age & $66(33-84)$ & $67(34-89)$ & .08 \\
Gender & & & .75 \\
$\quad$ Male & $169(44.1 \%)$ & $45(45.9 \%)$ & \\
Whole tumor size (cm) & $2.2(0.8-3.0)$ & $1.7(0.6-3.0)$ & $<.001$ \\
Solid tumor size (cm) & $1.5(0-3.0)$ & $0.5(0-3.0)$ & $<.001$ \\
SUVmax & $2.1(0-17)$ & $1.2(0-10)$ & $<.001$ \\
Side & & & .005 \\
$\quad$ Right & $261(68.4 \%)$ & $52(53.1 \%)$ & \\
Lobe & & & .001 \\
$\quad$ Upper & $200(52.2 \%)$ & $50(51.0 \%)$ & \\
$\quad$ Middle & $45(11.7 \%)$ & $0(0 \%)$ & \\
$\quad$ Lower & $138(36.0 \%)$ & $48(49.0 \%)$ & \\
Lymphatic invasion & $77(20.1 \%)$ & $6(6.1 \%)$ & .001 \\
Vascular invasion & $89(23.2 \%)$ & $6(6.1 \%)$ & $<.001$ \\
Pleural invasion & $51(13.3 \%)$ & $4(4.1 \%)$ & .008 \\
Lymph node metastasis & $44(11.5 \%)$ & $1(1.0 \%)$ & $<.001$ \\
\hline
\end{tabular}

SUVmax, Maximum standardized uptake value.

a coefficient that was calculated using logistic regression analysis, and the sum of these values was taken as the propensity score for individual patients. C statistic of variables was 0.819 (95\% confidence interval [CI], $0.776-0.863 ; P<.0001)$. After the calculation of their propensity scores, the subjects were divided into 3 groups according to tertile to compare characteristics between lobectomy and segmentectomy in each tertile. For matching, lobectomy and segmentectomy pairs with an equivalent propensity score were selected by a 1-to-1 match. Statistical Package for the Social Sciences (SPSS) software (version 10.5; SPSS Inc, Chicago, Ill) was used to statistically analyze the data.

\section{RESULTS}

Table 1 summarizes the characteristics of the 481 patients analyzed in this study. Of these, 383 patients underwent lobectomy and 98 patients underwent segmentectomy. There was no 30-day postoperative mortality in this population. The median follow-up period after surgery was 43.2 months, during which the tumor recurred in 50 patients. There were 20 local-only recurrences, including mediastinal lymph node metastasis, and 30 distant \pm local recurrences. Age and gender were not significantly different between patients who underwent lobectomy and those who underwent segmentectomy. Lobectomy was performed significantly more often for patients with large whole and solid tumor size, high SUVmax, pathologically invasive tumors (presence of lymphatic, vascular, or pleural invasion), and lymph node involvement. Tumor location was significantly different between patients who underwent lobectomy and those who underwent segmentectomy. Detailed procedures in segmentectomy were shown in Table 2.

Local recurrence occurred in 17 patients who underwent lobectomy ( 2 involving the bronchial stump, 1 involving the hilar lymph nodes, 11 involving the mediastinal lymph nodes, and 3 involving the pleura) and 3 patients who
TABLE 2. Details of segmentectomy $(n=98)$

\begin{tabular}{|c|c|c|c|}
\hline Site & No. & Site & No. \\
\hline \multicolumn{2}{|l|}{ Right } & \multicolumn{2}{|l|}{ Left } \\
\hline $\mathrm{S} 1$ & 4 & $\mathrm{~S} 1+2$ & 7 \\
\hline $\mathrm{S} 2$ & 12 & $\mathrm{~S} 3$ & 3 \\
\hline $\mathrm{S} 3$ & 3 & $\mathrm{~S} 1+2+3$ & 10 \\
\hline S6 & 23 & $\mathrm{~S} 1+2+3 \mathrm{c}$ & 1 \\
\hline S8 & 5 & $\mathrm{~S} 4$ & 2 \\
\hline $\mathrm{S} 7+8$ & 1 & S5 & 1 \\
\hline $\mathrm{S} 8+9$ & 3 & $\mathrm{~S} 4+5$ & 7 \\
\hline \multirow{4}{*}{$\mathrm{S} 7+8+9+10$} & 1 & S6 & 10 \\
\hline & & $\mathrm{S} 8$ & 1 \\
\hline & & S9 & 3 \\
\hline & & $\mathrm{S} 6+8+9+10$ & 1 \\
\hline
\end{tabular}

underwent segmentectomy (1 involving the residual lobe, 1 involving the surgical stump, and 1 involving the pleura).

Table 3 shows the multivariate analyses of distant and local RFS. Gender, solid tumor size, and SUVmax were significant independent prognostic factor for distant RFS, whereas whole tumor size was not. Regarding local RFS, solid tumor size and SUVmax were independent prognostic factors, but whole tumor size was not. RFS was not significantly different between patients who underwent lobectomy (3-year RFS, 87.3\%) compared with segmentectomy (3-year RFS, 91.4\%; hazard ratio [HR], 0.57; 95\% CI, $0.27-1.20 ; P=.14$, Figure $1, A$ ). OS was not significantly different between patients who underwent lobectomy (3-year OS, 94.1\%) compared with segmentectomy (3-year OS, 96.9\%; HR, 0.49; 95\% CI, 0.17-1.38; $P=.18$; Figure $1, B$ ).

After the calculation of the propensity score, the subjects were divided into 3 groups according to tertile (Table 4). The numbers of patients in tertiles 1,2, and 3 according to the operative procedures (lobectomy; segmentectomy) were 79 and 66, 118 and 27, and 141 and 5, respectively. Solid tumor size was smaller and SUVmax was lower in the lowest tertile group, indicating that segmentectomy trended to be performed in patients with a tumor of smaller solid tumor size and lower SUVmax. There were some differences in background characteristics, especially in the lowest tertile group. Therefore, we performed propensity score matching to compare the survival between lobectomy and segmentectomy groups.

When propensity score matching was used and variables such as age, gender, solid tumor size, SUVmax, side, and lobe were included, lobectomy and segmentectomy pairs were well matched (81 patients each) without significant differences in clinical and pathologic factors (Table 5).

Among propensity score-matched patients, no difference in RFS was identified between patients who underwent lobectomy (3-year RFS, 92.9\%) compared with segmentectomy (3-year RFS, 90.9\%; Figure 1, C). In addition, similar OSs were observed between patients who underwent 
TABLE 3. Multivariate analyses for distant or local RFS

\begin{tabular}{|c|c|c|}
\hline Variables & HR $(95 \%$ CI $)$ & $P$ value \\
\hline \multicolumn{3}{|l|}{ Multivariate analysis for distant RFS } \\
\hline \multicolumn{3}{|l|}{ Model 1} \\
\hline Age & $1.00(0.96-1.04)$ & .86 \\
\hline \multicolumn{3}{|l|}{ Gender } \\
\hline Male vs female & $2.62(1.15-5.95)$ & .022 \\
\hline Whole tumor size $(\mathrm{cm})$ & $1.17(0.60-2.27)$ & .65 \\
\hline SUVmax & $1.26(1.14-1.39)$ & $<.001$ \\
\hline \multicolumn{3}{|l|}{ Procedure } \\
\hline Lobectomy vs segmentectomy & $1.44(0.41-5.00)$ & .57 \\
\hline \multicolumn{3}{|l|}{ Model 2} \\
\hline Age & $1.00(0.96-1.03)$ & .80 \\
\hline \multicolumn{3}{|l|}{ Gender } \\
\hline Male vs female & $2.57(1.14-5.78)$ & .023 \\
\hline Solid tumor size $(\mathrm{cm})$ & $1.86(1.09-3.16)$ & .023 \\
\hline SUVmax & $1.19(1.06-1.34)$ & .003 \\
\hline \multicolumn{3}{|l|}{ Procedure } \\
\hline Lobectomy vs segmentectomy & $0.90(0.24-3.36)$ & .88 \\
\hline \multicolumn{3}{|l|}{ Multivariate analysis for local RFS } \\
\hline \multicolumn{3}{|l|}{ Model 1} \\
\hline Age & $1.04(0.99-1.10)$ & .15 \\
\hline \multicolumn{3}{|l|}{ Gender } \\
\hline Male vs female & $0.59(0.24-1.46)$ & .26 \\
\hline Whole tumor size $(\mathrm{cm})$ & $1.44(0.66-3.12)$ & .94 \\
\hline SUVmax & $1.17(1.03-1.33)$ & .015 \\
\hline \multicolumn{3}{|l|}{ Procedure } \\
\hline Lobectomy vs segmentectomy & $1.06(0.29-3.86)$ & .36 \\
\hline \multicolumn{3}{|l|}{ Model 2} \\
\hline Age & $1.04(0.98-1.09)$ & .19 \\
\hline \multicolumn{3}{|l|}{ Gender } \\
\hline Male vs female & $0.58(0.24-1.43)$ & .24 \\
\hline Solid tumor size $(\mathrm{cm})$ & $2.89(1.52-5.50)$ & .001 \\
\hline SUVmax & $1.09(0.94-1.27)$ & .26 \\
\hline \multicolumn{3}{|l|}{ Procedure } \\
\hline Lobectomy vs segmentectomy & $0.54(0.14-2.13)$ & .38 \\
\hline
\end{tabular}

RFS, Recurrence-free survival; $H R$, hazard ratio; $C I$, confidence interval; SUVmax, maximum standardized uptake value.

lobectomy (3-year OS, 93.2\%) compared with segmentectomy (3-year OS, 95.7\%; Figure 1,D).

\section{DISCUSSION}

The current study compared oncologic outcomes between patients who underwent lobectomy and segmentectomy for clinical stage IA lung adenocarcinoma. In all cohorts, when preoperative clinical factors were not adjusted, RFS and OS of the segmentectomy group were not significantly different from those of the lobectomy group. The survival curves of the segmentectomy group appeared to be better than those of the lobectomy group. However, each patient group was different in terms of solid tumor size and SUVmax, which could affect the patient's survival. ${ }^{8,9,13-16}$ In addition, the number of patients who had lymph node metastasis was inevitably larger in the lobectomy group than in the segmentectomy group, which also could affect the survival. To minimize patient selection bias, we used propensity score matching analyses. In the model that matched for potentially confounding variables such as age, gender, solid tumor size, SUVmax, tumor location, in lobectomy and segmentectomy pairs, there were no significant differences in clinical features or pathologic factors such as lymphatic, vascular, pleural invasion, or lymph node metastasis. Even in our matched model, RFS and OS in the segmentectomy group was similar to the lobectomy group, indicating that segmentectomy could be an optimal surgical procedure for clinical stage IA lung adenocarcinoma selected on the basis of HRCT and FDG-PET/CT.

The strength of this study was that variables such as findings from HRCT (solid tumor size) and FDG-PET (SUVmax) were included in the propensity score-matched analysis. We reported that solid tumor size on HRCT and SUVmax on FDG-PET/CT had higher predictive values with respect to pathologic invasiveness such as lymphatic, vascular, pleural invasion, and prognosis compared with whole tumor size. ${ }^{8,9}$ In addition, once matching for solid tumor size and SUVmax, pure solid tumor and solid tumor with GGO showed equivalent survivals. ${ }^{17}$ Indeed, whole tumor size was not an independent factor for distant or local RFS in this study, whereas solid tumor size and SUVmax were. Solid tumor size does represent tumor malignancy compared with whole tumor size. Therefore, we did not include whole tumor size in matching variables. Inasmuch as SUVmax on FDG-PET/CT was a prognostic indicator for lung adenocarcinoma, not for squamous cell carcinoma in our previous study, ${ }^{16}$ the database included only adenocarcinoma, which is a major histologic type for NSCLC. Although several studies have indicated equivalent survivals for segmentectomy and lobectomy in patients with clinical stage IA lung cancer, to our knowledge, this is the first study adjusting for preoperative HRCT and FDG-PET/CT findings, both of which should be considered when selecting patients for limited resections such as segmentectomy. Furthermore, we used an anthropomorphic body phantom to minimize the interinstitutional variability in SUV, which may be influenced by factors such as preparation procedures, scan acquisition, image reconstruction, and data analysis.

Most previous studies that showed favorable outcomes with segmentectomy indicated this procedure for T1 N0 M0 NSCLC of $2 \mathrm{~cm}$ or less. ${ }^{4-6}$ We included patients with a whole tumor size of 2 to $3 \mathrm{~cm}$ (ie, clinical T1b tumor) in this study. $\mathrm{We}^{9}$ have reported that patients with $\mathrm{T} 1 \mathrm{~b}$ lung adenocarcinomas selected on the basis of HRCT and FDG-PET/CT findings could be candidates for sublobar resection with a sufficient surgical margin. Inasmuch as clinical T1b N0 M0 lung adenocarcinomas occasionally show large GGO components and/or low SUVmax (signs of 
Recurrence-free survival

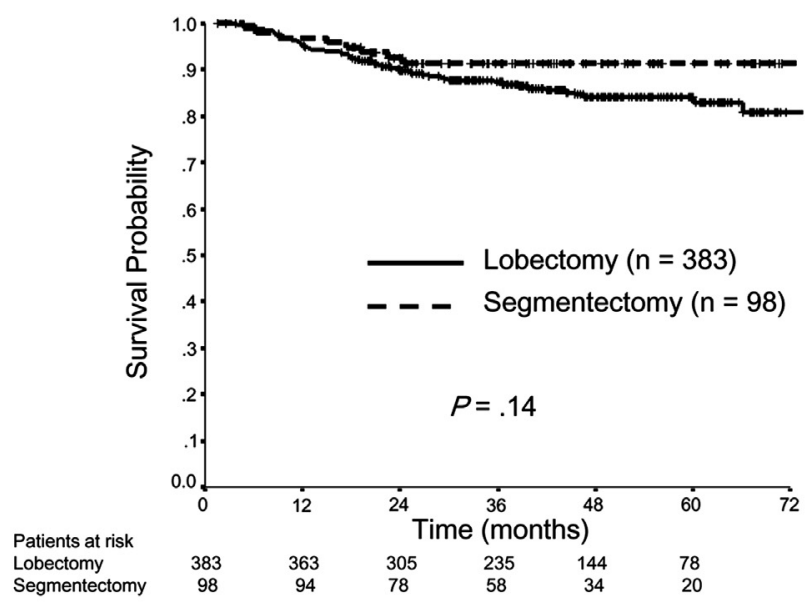

A

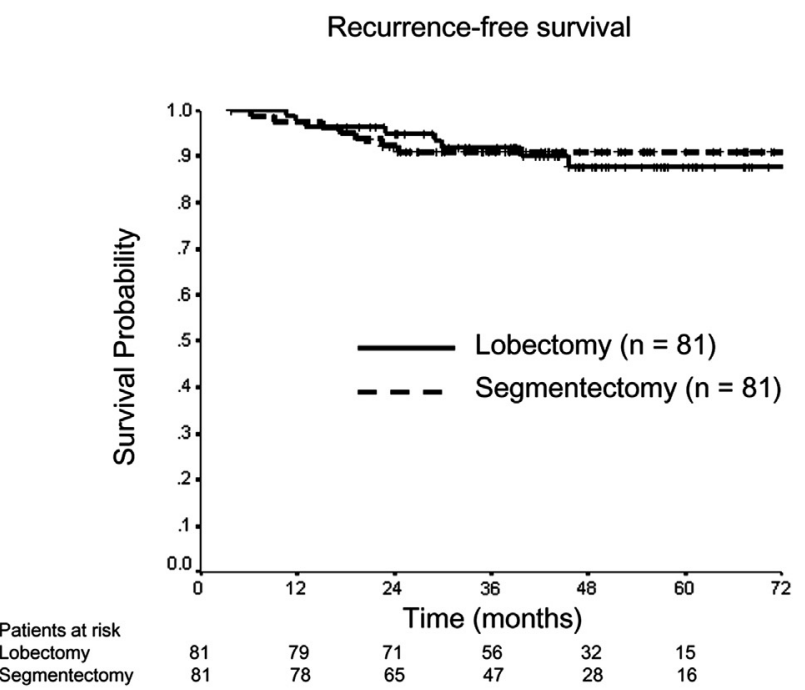

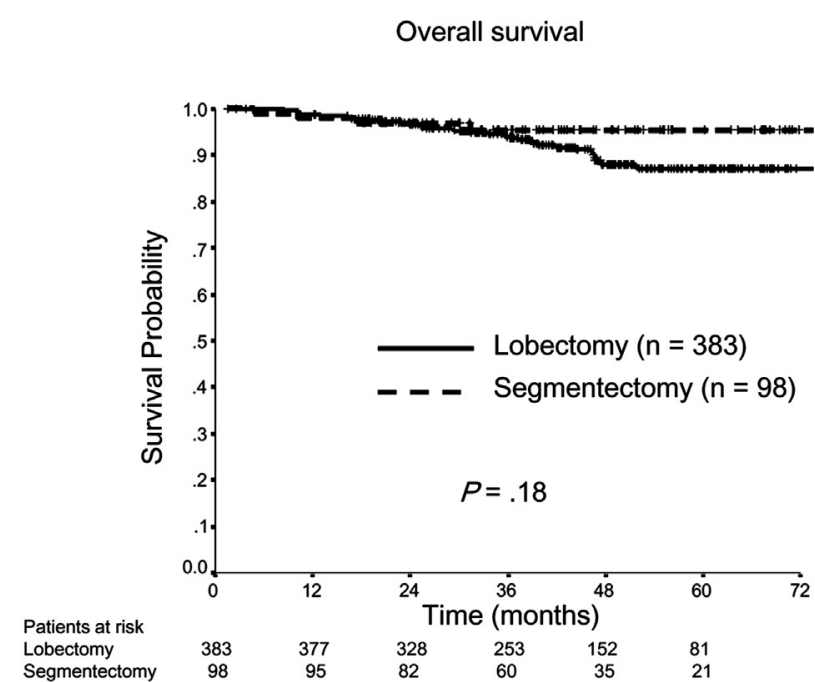

B

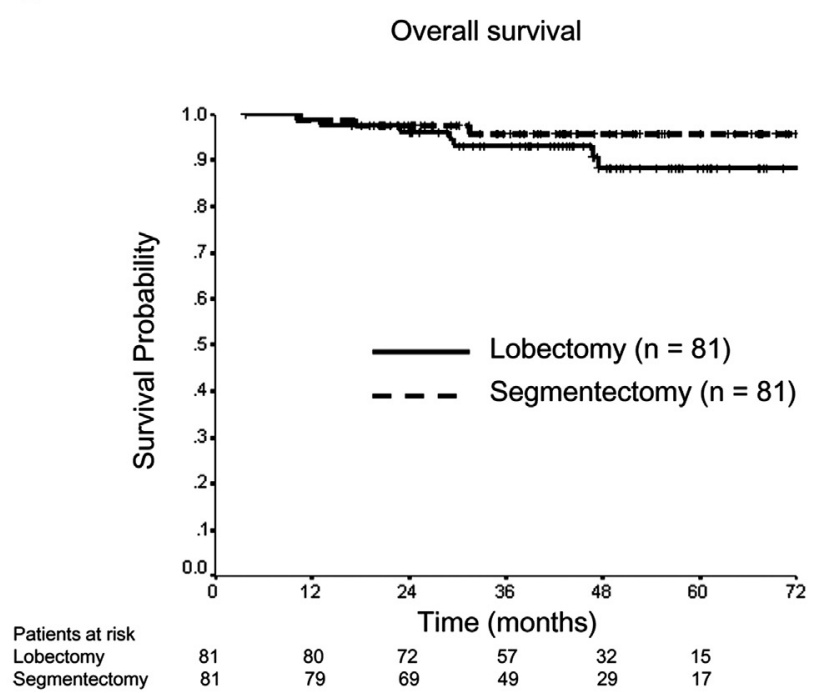

C

D

FIGURE 1. Recurrence-free survival (RFS) curves and overall survival (OS) curves for patients who underwent lobectomy and segmentectomy. A, In all cohorts, 3-year RFSs of 87.3\% (mean RFS, 66.8 months; 95\% confidence interval [CI], 64.6-69.4 months) and 91.4\% (mean RFS, 70.3 months; 95\% CI, 66.9-73.8 months) were identified for patients who underwent lobectomy and segmentectomy, respectively (hazard ratio [HR], 0.57; 95\% CI, 0.27-1.20; $P=.14$ ). B, In all cohorts, 3-year OSs of $94.1 \%$ (mean OS, 70.4 months; $95 \%$ CI, 68.7-72.1 months) and 96.9\% (mean OS, 72.9 months; $95 \%$ CI, $70.3-75.4$ months) were identified for patients who underwent lobectomy and segmentectomy, respectively (HR, $0.49 ; 95 \% \mathrm{CI}, 0.17-1.38 ; P=.18)$. C, In propensity score-matched patients, 3-year RFSs of 92.9\% (mean RFS, 68.6 months; 95\% CI, 64.9-72.2 months) and 90.9\% (mean RFS, 70.2 months; 95\% CI, 66.4-73.9 months) were identified for patients who underwent lobectomy and segmentectomy, respectively. D, In propensity score-matched patients, 3-year OSs of 93.2\% (mean OS, 69.3 months; 95\% CI, 65.8-72.7 months) and 95.7\% (mean OS, 73.2 months; 95\% CI, 70.6-75.8 months) were identified for patients who underwent lobectomy and segmentectomy, respectively.

low malignant behavior), such tumors could be treated with lesser resection. ${ }^{9}$

This study has several limitations. Because this study was retrospective, patients who underwent segmentectomy were possibly highly selective. In addition, we could not match intended procedures in the study because the database included only performed surgical procedures, not intended procedures, and patients with R1 or R2 resection were never included in the database. Most patients who underwent segmentectomy in this study tended to have relatively low-malignancy tumors, with small solid tumor size and/ or low SUVmax, and thus low pathologic invasiveness. The present study revealed that large solid tumor size on HRCT and high SUVmax on FDG-PET/CT were significantly associated with both local and distant recurrences. The outcome of segmentectomy for relatively highmalignancy clinical stage IA lung adenocarcinomas with large solid tumor size and high SUVmax is unclear. 
TABLE 4. Patient characteristics divided into 3 groups according to tertile based on the propensity score

\begin{tabular}{|c|c|c|c|c|c|c|c|c|c|}
\hline & \multicolumn{3}{|c|}{ Tertile 1} & \multicolumn{3}{|c|}{ Tertile 2} & \multicolumn{3}{|c|}{ Tertile 3} \\
\hline & $L(n=79)$ & $S(n=66)$ & $P$ value & $L(n=118)$ & $\mathbf{S}(\mathbf{n}=\mathbf{2 7})$ & $P$ value & $L(n=141)$ & $\mathbf{S}(\mathbf{n}=\mathbf{5})$ & $P$ value \\
\hline Age & $68(48-82)$ & $68.5(42-89)$ & .53 & $65(40-83)$ & $65(34-86)$ & .92 & $65(33-84)$ & $64(53-83)$ & .5 \\
\hline Gender & & & .87 & & & .28 & & & 1.0 \\
\hline Male & $36(45.6 \%)$ & $29(43.9 \%)$ & & $46(39.0 \%)$ & $14(51.9 \%)$ & & $65(46.1 \%)$ & $2(40.0 \%)$ & \\
\hline Whole tumor size $(\mathrm{cm})$ & $2.0(0.8-3.0)$ & $1.7(0.9-3.0)$ & .01 & $1.8(1.0-3.0)$ & $1.6(0.6-2.9)$ & .048 & $2.5(1.2-3.0)$ & $2.4(1.5-3.0)$ & .51 \\
\hline Solid tumor size $(\mathrm{cm})$ & $0.5(0-2.0)$ & $0.3(0-1.0)$ & .056 & $1.4(0-2.0)$ & $1.2(0-2.0)$ & .03 & $2.3(1.0-3.0)$ & $2.2(1.0-3.0)$ & .71 \\
\hline SUVmax & $1.2(0-4.9)$ & $1.0(0-4.1)$ & .002 & $1.9(0.6-8.3)$ & $1.9(0.4-9.8)$ & .77 & $3.9(0.7-16.9)$ & $2.1(1.5-4.3)$ & .13 \\
\hline Side & & & .41 & & & 1.0 & & & 1.0 \\
\hline Right & $44(55.7 \%)$ & $32(48.5 \%)$ & & $69(58.5 \%)$ & $16(59.3 \%)$ & & $103(73.0 \%)$ & $4(80.0 \%)$ & \\
\hline Lobe & & & .51 & & & .53 & & & 1.0 \\
\hline Upper & $41(51.9 \%)$ & $30(45.5 \%)$ & & $66(55.9 \%)$ & $17(63.0 \%)$ & & $93(66.0 \%)$ & $3(60.0 \%)$ & \\
\hline Lower & $38(48.1 \%)$ & $36(54.5 \%)$ & & $52(44.1 \%)$ & $10(37.0 \%)$ & & $48(34.0 \%)$ & $2(40.0 \%)$ & \\
\hline
\end{tabular}

$L$, Lobectomy; $S$, segmentectomy; SUVmax, maximum standardized uptake value.

Although surgical procedure did not correlate with local or distant recurrence in this study, segmentectomy for such tumors (ie, with large solid tumor size or high SUVmax) should be carefully considered. A clinical trial is being conducted by the Japanese Clinical Oncology Group/West Japan Oncology Group (JCOG0802/WJOG4607L), which aims to compare the surgical results between lobectomy and segmentectomy for T1 N0 M0 NSCLC measuring 2 $\mathrm{cm}$ or less. ${ }^{18}$ This prospective study includes patients with radiologically invasive tumors, such as solid dominant tumors, that have large solid tumor size on HRCT. The results of this trial may provide an important insight into this issue.

Segmentectomy is beneficial because it preserves lung function. Although the database used in this study did not incorporate lung function data, several reports have

TABLE 5. Propensity score-matched comparison of clinical and pathologic factors between patients who underwent lobectomy and segmentectomy

\begin{tabular}{lccc}
\hline & $\begin{array}{c}\text { Lobectomy } \\
(\mathbf{n}=\mathbf{8 1})\end{array}$ & $\begin{array}{c}\text { Segmentectomy } \\
(\mathbf{n}=\mathbf{8 1})\end{array}$ & $\boldsymbol{P}$ value \\
\hline Clinical factors & & & \\
$\quad$ Age & $66(48-82)$ & $65(34-86)$ & .68 \\
Gender & & & .74 \\
$\quad$ Male & $37(45.6 \%)$ & $34(42.0 \%)$ & \\
Whole tumor size $(\mathrm{cm})$ & $2.0(1.0-3.0)$ & $1.7(0.6-3.0)$ & .11 \\
Solid tumor size $(\mathrm{cm})$ & $0.7(0-2.0)$ & $0.8(0-3.0)$ & .17 \\
SUVmax & $1.4(0-7.0)$ & $1.2(0-9.8)$ & .23 \\
Side & & & .63 \\
$\quad$ Right & $33(40.7 \%)$ & $37(45.6 \%)$ & \\
Lobe & & & .23 \\
$\quad$ Upper & $51(63.0 \%)$ & $43(53.1 \%)$ & \\
$\quad$ Lower & $30(37.0 \%)$ & $38(46.9 \%)$ & \\
Pathologic factors & & & .42 \\
$\quad$ Lymphatic invasion & $10(12.3 \%)$ & $6(7.4 \%)$ & .45 \\
$\quad$ Vascular invasion & $6(7.4 \%)$ & $6(7.4 \%)$ & .63 \\
$\quad$ Pleural invasion & $7(8.6 \%)$ & $4(4.9 \%)$ & .45 \\
Lymph node metastasis & $3(3.7 \%)$ & $1(1.2 \%)$ & .63 \\
\hline
\end{tabular}

SUVmax, Maximum standardized uptake value. demonstrated that segmentectomy has functional advantages over lobectomy. ${ }^{5,19,20}$ If similar oncologic outcomes are expected, segmentectomy should be considered for patients with clinical stage IA lung adenocarcinoma.

In conclusion, the oncologic outcomes of segmentectomy are similar to those of standard lobectomy for patients with clinical stage IA lung adecnocarcinoma, as determined by the matched model adjusting for preoperative clinical factors such as HRCT and FDG-PET/CT findings. Segmentectomy could be favorable for selective patients with stage IA lung adenocarcinoma.

\section{References}

1. Ginsberg RH, Rubinstein LV. Randomized trial of lobectomy versus limited resection for T1N0 non-small cell lung cancer. Lung Cancer Study Group. Ann Thorac Surg. 1995;60:615-23.

2. Whitson BA, Groth SS, Andrade RS, Maddaus MA, Habermann EB, D'Cunha J. Survival after lobectomy versus segmentectomy for stage I non-small cell lung cancer: a population-based analysis. Ann Thorac Surg. 2011;92:1943-50.

3. Jensik RJ, Faber LP, Milloy FJ, Monson DO. Segmental resection for lung carcinoma: a fifteen-year experience. J Thorac Cardiovasc Surg. 1973;66:563-72.

4. Okada M, Yoshikawa K, Hatta T, Tsubota N. Is segmentectomy with lymph node assessment an alternative to lobectomy for non-small cell lung cancer of $2 \mathrm{~cm}$ or smaller? Ann Thorac Surg. 2001;71:956-61.

5. Yoshikawa K, Tsubota N, Kodama K, Ayabe H, Taki T, Mori T. Prospective study of extended segmentectomy for small lung tumors: the final report. Ann Thorac Surg. 2002;73:1055-9.

6. Okada M, Koike T, Higashiyama M, Yamato Y, Kodama K, Tsubota N. Radical sublobar resection for small-sized non-small cell lung cancer: a multicenter study. J Thorac Cardiovasc Surg. 2006;132:769-75.

7. Okada M, Tsutani Y, Ikeda T, Misumi K, Matsumoto K, Yoshimura M, et al. Radical hybrid video-assisted thoracic segmentectomy: long-term results of minimally invasive anatomical sublobar resection for treating lung cancer. Interact Cardiovasc Thorac Surg. 2012;14:5-11.

8. Tsutani Y, Miyata Y, Nakayama H, Okumura S, Adachi S, Yoshimura M, et al. Prognostic significance of using solid versus whole tumor size on highresolution computed tomography for predicting the pathological malignant grade of tumors in clinical stage IA lung adenocarcinoma: a multicenter study. J Thorac Cardiovasc Surg. 2012;143:607-12.

9. Tsutani Y, Miyata Y, Nakayama H, Okumura S, Adachi S, Yoshimura M, et al. Prediction of pathological node-negative clinical stage IA lung adenocarcinoma for optimal candidates undergoing sublobar resection. J Thorac Cardiovasc Surg. 2012;144:1365-71

10. Goldstraw P, Crowley J, Chansky K, Giroux DJ, Groome PA, Rami-Porta R, et al. International Association for the Study of Lung Cancer International Staging Committee; Participating Institutions. The IASLC Lung Cancer Staging Project: 
proposals for the revision of the TNM stage groupings in the forthcoming (seventh) edition of the TNM Classification of Malignant Tumours. $J$ Thorac Oncol. 2007;2:706-14.

11. Delbeke D, Coleman RE, Guiberteau MJ, Brown ML, Royal HD, Siegel BA, et al. Procedure guideline for tumor imaging with 18F-FDG PET/CT 1.0. J Nucl Med. 2006;47:885-95.

12. Mawlawi O, Podoloff DA, Kohlmyer S, Williams JJ, Stearns CW, Culp RF, et al. Performance characteristics of a newly developed PET/CT scanner using NEMA standards in 2D and 3D modes. J Nucl Med. 2004;45:1734-42.

13. Nakayama H, Okumura S, Daisaki H, Kato Y, Uehara H, Adachi S, et al. Value of integrated positron emission tomography revised using a phantom study to evaluate malignancy grade of lung adenocarcinoma. Cancer. 2010; 116:3170-7.

14. Okada M, Nakayama H, Okumura S, Daisaki H, Adachi S, Yoshimura M, et al. Multicenter analysis of high-resolution computed tomography and positron emission tomography/computed tomography findings to choose therapeutic strategies for clinical stage IA lung adenocarcinoma. J Thorac Cardiovasc Surg. 2011;141:1384-91.

15. Okada M, Tauchi S, Iwanaga K, Mimura T, Kitamura Y, Watanabe H, et al. Associations among bronchioloalveolar carcinoma components, positron emission tomographic and computed tomographic findings, and malignant behavior in small lung adenocarcinomas. J Thorac Cardiovasc Surg. 2007;133: 1448-54.

16. Tsutani Y, Miyata Y, Misumi K, Ikeda T, Mimura T, Hihara J, et al. Difference in prognostic significance of maximum standardized uptake value on [18F]-fluoro2-deoxyglucose positron emission tomography between adenocarcinoma and squamous cell carcinoma of the lung. Jpn J Clin Oncol. 2011;41:890-6.

17. Tsutani Y, Miyata Y, Yamanaka T, Nakayama H, Okumura S, Adachi S, et al. Solid tumors versus mixed tumors with a ground-glass opacity component in patients with clinical stage IA lung adenocarcinoma: prognostic comparison using high-resolution computed tomography findings. J Thorac Cardiovasc Surg. 2013;146:17-23.

18. Nakamura K, Saji H, Nakajima R, Okada M, Asamura H, Shibata T, et al. A phase III randomized trial of lobectomy versus limited resection for small-sized peripheral non-small cell lung cancer (JCOG0802/WJOG4607L). Jpn J Clin Oncol. 2010;40:271-4.

19. Keenan RJ, Landreneau RJ, Maley RH, Singh D, Macherey R, Bartley S, et al. Segmental resection spares pulmonary function in patients with stage I lung cancer. Ann Thorac Surg. 2004;78:228-33.

20. Harada H, Okada M, Sakamoto T, Matsuoka H, Tsubota N. Functional advantage after radical segmentectomy versus lobectomy for lung cancer. Ann Thorac Surg. 2005;80:2041-5. 